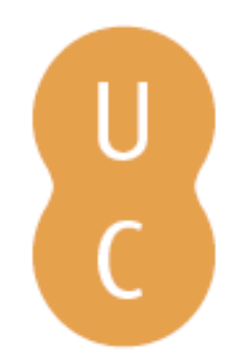

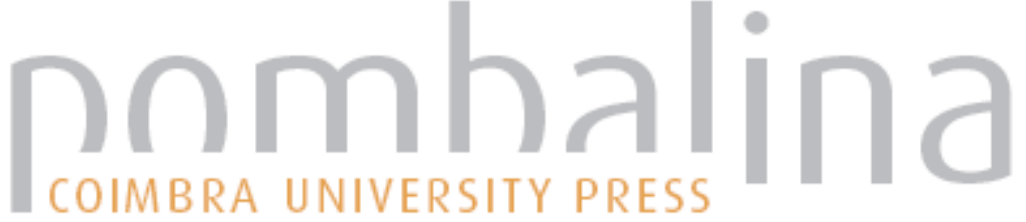

\section{Otimização do planeamento inverso de tratamentos em radioterapia}

Autor(es): $\quad$ Rocha, H.; Dias, J. M.; Ferreira, B. C.; Lopes, M. C.

Publicado por: Imprensa da Universidade de Coimbra

URL

persistente:

URI:http://hdl.handle.net/10316.2/35939

DOI:

DOI:http://dx.doi.org/10.14195/978-989-26-0738-2_17

Accessed : $\quad$ 26-Apr-2023 02:50:14

A navegação consulta e descarregamento dos títulos inseridos nas Bibliotecas Digitais UC Digitalis, UC Pombalina e UC Impactum, pressupõem a aceitação plena e sem reservas dos Termos e Condições de Uso destas Bibliotecas Digitais, disponíveis em https://digitalis.uc.pt/pt-pt/termos.

Conforme exposto nos referidos Termos e Condições de Uso, o descarregamento de títulos de acesso restrito requer uma licença válida de autorização devendo o utilizador aceder ao(s) documento(s) a partir de um endereço de IP da instituição detentora da supramencionada licença.

Ao utilizador é apenas permitido o descarregamento para uso pessoal, pelo que o emprego do(s) título(s) descarregado(s) para outro fim, designadamente comercial, carece de autorização do respetivo autor ou editor da obra.

Na medida em que todas as obras da UC Digitalis se encontram protegidas pelo Código do Direito de Autor e Direitos Conexos e demais legislação aplicável, toda a cópia, parcial ou total, deste documento, nos casos em que é legalmente admitida, deverá conter ou fazer-se acompanhar por este aviso. 


\section{INVESTIGAÇÃO OPERACIONAL EM AÇÃO \\ CASOS DE APLICAÇÃO}

RUI CARVALHO OLIVEIRA JOSÉ SOEIRO FERREIRA (EDITORES)

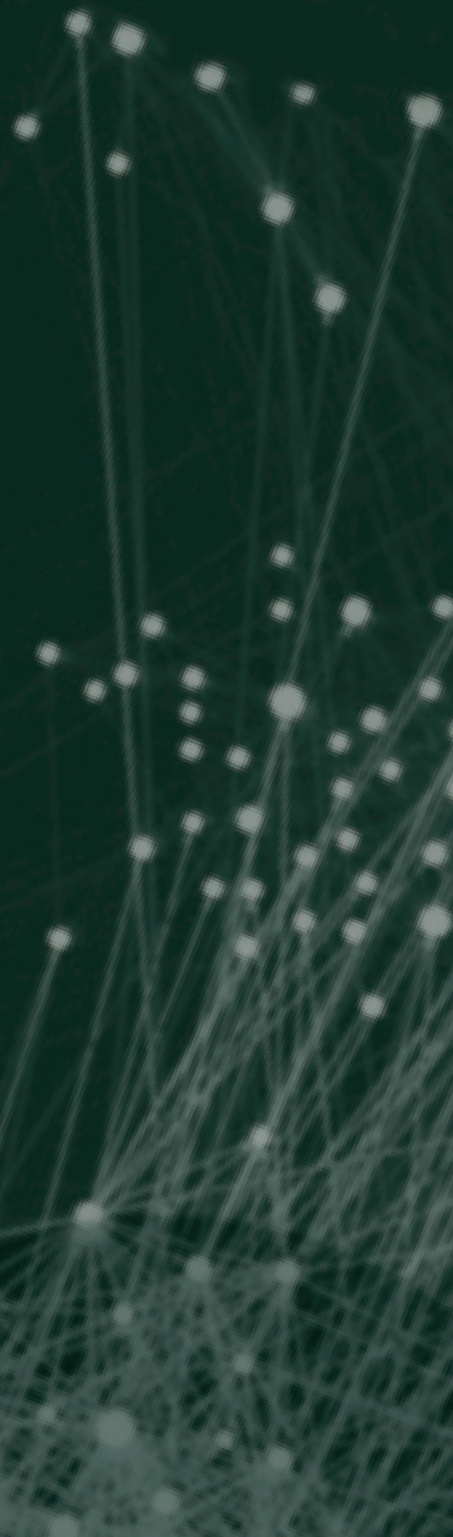




\title{
CASO 17
}

\section{OTIMIZAÇÃO DO PLANEAMENTO INVERSO DE TRATAMENTOS EM RADIOTERAPIA}

\author{
H. Rocha \\ INESC-Coimbra, Coimbra, Portugal \\ hrocha@mat.uc.pt \\ J.M. Dias \\ INESC-Coimbra, Coimbra, Portugal \\ Faculdade de Economia, Universidade de Coimbra, Coimbra \\ joana@fe.uc.pt \\ B.C. Ferreira \\ I3N, Departamento de Física, Universidade de Aveiro, Aveiro, Portugal \\ Serviço de Física Médica, IPOC-FG, EPE, Coimbra, Portugal \\ brigida@ua.pt \\ M.C. Lopes \\ I3N, Departamento de Física, Universidade de Aveiro, Aveiro, Portugal \\ Serviço de Física Médica, IPOC-FG, EPE, Coimbra, Portugal \\ mclopes@ipocoimbra.min-saude.pt
}




\section{RESUMO}

O número de casos de cancro continua a crescer à escala planetária. Existem vários tipos de tratamento usados habitualmente, dependendo do tipo e fase do cancro, incluindo cirurgia, radioterapia, quimioterapia, imunoterapia, etc. Neste trabalho, vamos focar a otimização do planeamento de tratamentos em radioterapia, em particular de tratamentos em radioterapia com intensidade modulada (IMRT), onde a otimização tem um papel importante na melhoria da qualidade dos tratamentos. O contínuo desenvolvimento de novas máquinas de tratamento contribui para a melhoria da precisão e maior controlo da irradiação dos pacientes. A investigação operacional tem acompanhado a evolução destas máquinas e tecnologias, e tem feito contribuições significativas na melhoria do planeamento dos tratamentos em radioterapia, sendo os tratamentos em IMRT um bom exemplo disso. Devido à complexidade dos problemas de otimização do planeamento de tratamentos em IMRT, estes são usualmente divididos em três sub-problemas de menor dimensão que podem ser resolvidos sequencialmente: problema de otimização angular, problema de otimização das intensidades e problema de sequenciação das folhas. O primeiro destes sub-problemas consiste na escolha das melhores direções de incidência da radiação, encontrando-se ainda por resolver de forma satisfatória. Na prática clínica, a maior parte das vezes, as direções de incidência da radiação continuam a ser escolhidas manualmente, com base essencialmente na experiência do físico-médico, sem garantia da obtenção dos planos de tratamento de melhor qualidade. A abordagem que usamos considera os problemas de otimização angular e otimização das intensidades de forma integrada. Vamos demostrar que a investigação operacional tem um papel importante na obtenção de melhores planos de tratamento, focando-nos principalmente na aproximação por nós sugerida para o problema de otimização angular.

\section{PALAVRAS-CHAVE}

Radioterapia; Otimização; Planeamento inverso. 


\section{Introdução}

O número de casos de cancro continua a crescer à escala planetária. Um estudo da American Cancer Society (2010) estima que mais de 33 mil novos casos de cancro surjam diariamente. O mesmo estudo prevê que, em 2050, existam mais de 27 milhões de pessoas com cancro. Em 2012, o número estimado de novos casos nos EUA atinge os 1.5 milhões, sendo esperado que mais de meio milhão de norte-americanos morram de cancro, mais de 1500 por dia. Na Europa, com quase 500 milhões de habitantes, estima-se que sejam diagnosticados, por ano, cerca de 2 milhões de novos casos. Em Portugal, não existem números oficiais, devido à ausência de um registo sistematizado de ocorrências. No entanto, a Liga Portuguesa Contra o Cancro estima que, por ano, morram 42 mil pessoas com cancro em Portugal e deixa ainda o alerta relativo à duplicação de novos casos até 2030.

A prevenção e o rápido diagnóstico são as melhores armas contra o cancro. Para os cancros diagnosticados nos EUA entre 1996 e 2004, a taxa de sobrevivência a 5 anos é de 66\%, melhor do que os 50\% verificados entre 1975 e 1977 (American Cancer Society, 2010). Esta melhoria reflete o esforço em prevenção e rápido diagnóstico, mas também a melhoria contínua da qualidade dos tratamentos oferecidos para os diferentes tipos de cancro. Existem várias possibilidades de tratamento usados habitualmente, dependendo do tipo e fase do cancro, e incluem cirurgia, radioterapia, quimioterapia, imunoterapia, etc. Uma combinação destes tratamentos é frequentemente usada para obter os melhores resultados. Neste trabalho, vamos focar-nos no planeamento de tratamentos em radioterapia, em particular com intensidade modulada (IMRT), onde a otimização tem um papel importante na melhoria da qualidade dos tratamentos. Os termos radioterapia e otimização estão relacionados entre si desde 1959 (Carlson \& Morkovin, 1959). A interação entre a investigação operacional e a física médica teve o seu primeiro passo em 1968 com o uso do primeiro modelo de programação linear para ajudar ao desenho de modelos em radioterapia (Bahr et al, 1968). Apareceu deste modo uma área multidisciplinar florescente e de importância crescente. Muitos artigos de revisão foram já produzidos nesta área multidisciplinar (e.g., Bortfeld, 2006; 
Censor, 2003; Ehrgott et al, 2008; Holder \& Salter, 2004; Rocha \& Dias, 2009) o que é mais uma prova do interesse que este tema tem suscitado.

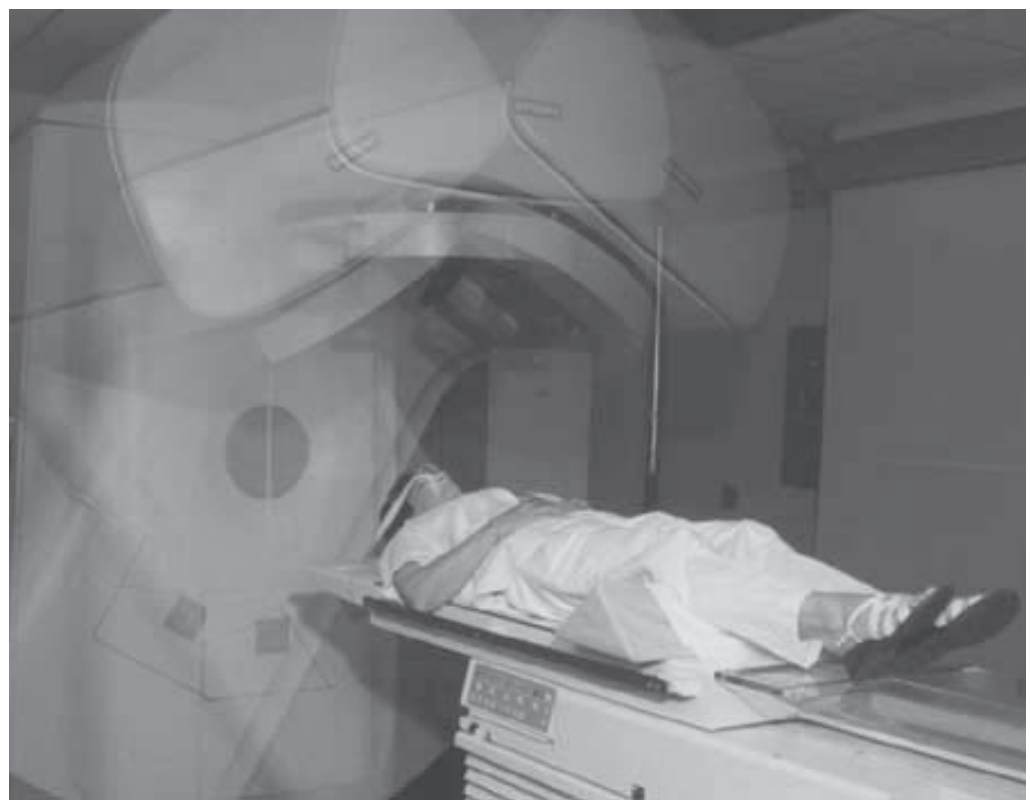

Figura 1: Acelerador linear (linac) irradiando em torno do paciente (RadiologyInfo, 2012).

O objetivo da radioterapia é administrar a dose de radiação prescrita pelo médico ao volume delineado (o volume do tumor mais uma margem de segurança), designado por PTV, de forma a matar as células cancerosas minimizando os danos nos tecidos e órgãos circundantes. A radioterapia baseia-se no facto de as células cancerosas se reproduzirem muito mais rapidamente do que as células saudáveis e serem incapazes de sobreviver quando danificadas por radiação. Como tal, o objetivo do tratamento é emitir radiação suficiente de forma a matar as células cancerosas mas não demasiada que comprometa a capacidade das células saudáveis em sobreviver. Tipicamente, a radiação é gerada por um acelerador linear (linac) que roda em torno de um eixo central, e é emitida com o paciente imobilizado numa mesa de tratamentos que também pode rodar (Figura 1). A combinação destes dois movimentos de rotação permite uma irradiação a partir de qualquer ângulo em torno do PTV. Contudo, como forma de reduzir um problema já complexo, o 
uso de ângulos coplanares continua a ser predominante, pertencendo os ângulos habitualmente usados ao plano de rotação do linac em torno do paciente. Na prática clínica, a maior parte das vezes, o número de ângulos é definido a priori e as direções de incidência da radiação são selecionadas manualmente pelo físico-médico, que confia essencialmente na sua experiência. A literatura apresenta, no entanto, evidência de que direções de incidência da radiação apropriadas podem levar a melhorias na qualidade dos tratamentos (Das \& Marks, 1997; Liu et al, 2006; Rowbottom et al,. 1998). O contínuo desenvolvimento de novas máquinas contribui para a melhoria da precisão e maior controlo da irradiação. A investigação operacional tem acompanhado a evolução das máquinas e tecnologia e tem feito contribuições significativas na melhoria do planeamento dos tratamentos em radioterapia (Bahr et al, 1968; Ferris et al, 2003a; Ferris et al, 2003b; Lim et al, 2007; Lim \& Lee, 2007; Romeijin et al, 2006), sendo os tratamentos em IMRT um bom exemplo disso.

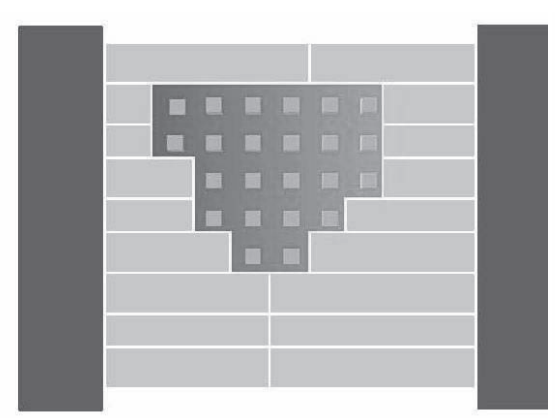

(a)

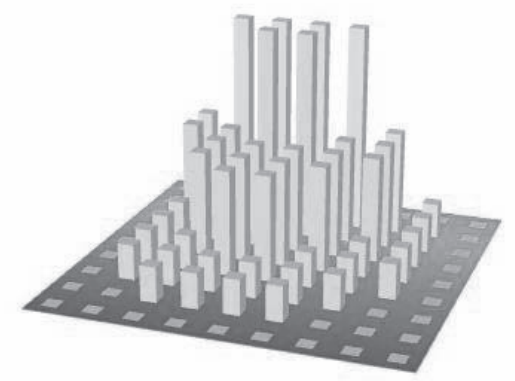

(b)

Figura 2: Ilustração de um MLC (com 9 pares de folhas) - 2(a) e ilustração de um mapa de intensidades (9X9) - 2(b).

A otimização do planeamento de um tratamento pode ser interpretada como a seleção ótima de uma dada configuração dos parâmetros do acelerador linear, entre um conjunto de possíveis configurações (soluções admissíveis). Dada a complexidade do problema, muitas vezes o planeamento é feito através de um processo de tentativa e erro, designado por planeamento direto. No planeamento direto, uma dada configuração é definida e as doses de 
radioterapia que vão ser absorvidas pelo organismo do doente são calculadas. Se estas doses estiverem de acordo com a prescrição médica, o planeamento termina. Em caso contrário, o processo continua através da alteração manual de alguns dos parâmetros da configuração. O planeamento inverso consiste em calcular a configuração ótima, dadas as doses prescritas, usando modelos e programas de otimização. O planeamento inverso permite modelar problemas de planeamento de tratamentos altamente complexos e a otimização tem um papel fundamental no sucesso deste procedimento. Um tipo importante de tratamentos com planeamento inverso são os tratamentos em IMRT, onde o feixe de radiação é modulado por um colimador multifolhas (MLC) (Figura 2(a)), o que permite transformar o feixe de radiação em pequenos sub-feixes de intensidades independentes (Figura 2(b)). Apesar da ilustração na Figura 2(b), os sub-feixes não existem fisicamente, sendo gerados pelo movimento das folhas do MLC que bloqueiam parte do feixe durante porções do tempo de irradiação. Uma forma comum de resolver os problemas de planeamento inverso na otimização de tratamentos em IMRT é usar uma aproximação baseada nos sub-feixes de radiação, o que origina problemas de programação de grande dimensão com centenas de variáveis e dezenas de milhar de restrições. A qualidade do plano de tratamento, dependendo dos modelos de programação e correspondentes métodos de resolução, determina a eficácia do tratamento. Devido à complexidade de tais problemas de otimização, estes são usualmente divididos em três sub-problemas de menor dimensão, que podem ser resolvidos sequencialmente: problema de otimização angular, problema de otimização das intensidades e problema de sequenciação das folhas. A abordagem que usamos considera os problemas de otimização angular e otimização das intensidades de forma integrada.

O problema de otimização angular tenta encontrar o número ótimo de ângulos (direções) a utilizar no tratamento e a sua localização. Muitas vezes considera-se o número de ângulos fixo a priori, limitando-se a otimização à procura dos melhores ângulos dentro de todas as combinações possíveis. O problema de otimização das intensidades considera os ângulos já definidos, e tenta otimizar as intensidades dos feixes de radiação para cada direção de 
incidência. O problema de sequenciação das folhas determina de que forma será efetuado o movimento das folhas do MLC, tendo em conta as restrições físicas do equipamento, por forma a se obterem as intensidades de radiação desejadas. A maior parte dos esforços dos investigadores em otimização de tratamentos em IMRT foram dedicados à otimização das intensidades (Craft, 2007), sendo os ângulos de irradiação determinados pelo físico-médico. Comparativamente menos esforço de investigação tem sido dedicado ao problema de otimização angular (Ehrgott \& Johnston, 2003). Este é um problema de otimização muito difícil, ainda em aberto, visto ser um problema altamente não convexo com muitos mínimos locais. Neste trabalho, vamos demonstrar que a investigação operacional tem um papel importante na obtenção de planos de tratamento de maior qualidade, focando a aproximação por nós sugerida para o problema de otimização angular, e descrevendo muito sucintamente os outros sub-problemas. Para tal, vamos usar dois exemplos clínicos de casos de tumores de cabeça e pescoço tratados retrospectivamente no Instituto Português de Oncologia Francisco Gentil de Coimbra (IPOC), instituição com quem temos colaborado. É importante salientar que as melhorias obtidas nos planos de tratamento otimizados se refletem numa efetiva melhoria da qualidade de vida dos pacientes. Na próxima secção vamos descrever o problema de otimização das intensidades, necessário para a nossa formulação do problema de geometria angular. Na secção 3 apresentamos o problema de otimização angular, a formulação e método de resolução por nós sugeridos. Os resultados numéricos para os casos de tumores de cabeça e pescoço descritos na secção 4 são apresentados na secção 5 . Na última secção temos a conclusão.

\section{Problema de otimização das intensidades}

Considerando que os ângulos (ou direções) de irradiação estão já definidos, o paciente é tratado usando um plano ótimo obtido após a resolução do problema de otimização das intensidades - o problema de determinar a intensidade ótima de cada um dos sub-feixes para cada um dos feixes fixos correspondentes às direções de irradiação já definidas. Muitos modelos 
matemáticos e algoritmos têm sido propostos para este problema, incluindo modelos lineares (e.g., Romeijn et al, 2005; Romeijn et al, 2003), modelos lineares inteiros mistos (e.g., Lee et al, 2003; Preciado-Walters et al, 2006), modelos não lineares (e.g., Aleman et al, 2008; Spirou \& Chui, 1998) e modelos multiobjetivo (e.g., Craft et al, 2006; Romeijn et al, 2004).

A distribuição de dose depositada no paciente, medida em Grays (Gy), precisa de ser aferida com precisão de forma a resolver o problema de otimização das intensidades. O volume de cada estrutura é discretizado em voxeis (pequenos elementos de volume) e a dose é calculada para cada voxel usando o princípio da superposição, i.e., considerando a contribuição de cada sub-feixe. Tipicamente, uma matriz de dose $D$ é construída a partir do conjunto de todas as intensidades dos sub-feixes, indexando as linhas de $D$ a cada voxel e as colunas de $D$ a cada sub-feixe, i.e., o número de linhas de $D$ é igual ao número total de voxeis $\left(N_{v}\right)$ e o número de colunas é igual ao número total de sub-feixes $\left(N_{b}\right)$ de todos os feixes de radiação considerados. O cálculo preciso da matriz $D$ é alvo contínuo de investigação, existindo diferentes algoritmos de dose, sendo o Monte Carlo a técnica padrão. Por razões de economia de tempo, utilizamos um algoritmo menos preciso mas mais rápido (pencil beam) para calcular a matriz $D$, que se mantém constante ao longo do processo de otimização para cada conjunto de direções de irradiação. Portanto, usando formato matricial, podemos dizer que a dose total recebida pelo voxel $i$ é dada por $\sum_{j=1}^{N_{b}} D_{i j} w_{j}$, com $w_{j}$ a intensidade do sub-feixe $j$. Usualmente, o número de voxeis considerados atinge as dezenas de milhar, portanto o número de linhas da matriz de dose é dessa magnitude. O tamanho da matriz $D$ origina problemas de grande dimensão, sendo uma das principais razões para a dificuldade em resolver o problema de otimização das intensidades. Uma das estratégias para reduzir a dimensão dos problemas de otimização e simultaneamente o tempo total de cálculo é usar sampling (Rocha et al, 2011). A agregação de voxeis em certas estruturas (sobretudo nas células do tecido normal), origina uma redução da dimensão do problema, o que conduz a uma resolução mais rápida do mesmo.

As primeiras tentativas de abordagem ao problema de otimização das 
intensidades fizeram uso de modelos de programação linear. Algumas das razões para o uso destes modelos incluem o facto de a dose ser depositada de forma linear, os modelos lineares serem fáceis de implementar, usados extensivamente, rápidos a obter soluções ótimas e resolúveis mesmo para modelos de muito grande dimensão. Dada uma prescrição com limites inferior e superior para a dose do PTV, limites superiores para as doses dos órgãos de risco (OARs) em torno do tumor, limite superior para a dose do restante tecido de células normais (NT) e um valor máximo para as intensidades dos sub-feixes de radiação (M), a maioria das formulações dos modelos de programação linear pertencem a uma classe de modelos de otimização com restrições, tal que uma função objetivo é minimizada enquanto as doses permanecem dentro dos limites impostos:

$$
\begin{aligned}
\min _{w} f(D) & \\
\text { t.q. } \quad L B_{P T V} \leq & \sum_{j=1}^{N_{b}} D_{i j} w_{j} \leq U B_{P T V}, \forall i \in P T V, \\
& \sum_{j=1}^{N_{b}} D_{i j} w_{j} \leq U B_{O A R}, \forall i \in O A R, \\
& \sum_{j=1}^{N_{b}} D_{i j} w_{j} \leq U B_{N T}, \forall i \in N T, \\
& \leq w_{j} \leq M, j=1, \ldots, N_{b} .
\end{aligned}
$$

Outras restrições poderiam ser incluídas dependendo do que for pretendido pelo físico-médico. Uma variedade de diferentes modelos pode ser formulada combinando diferentes restrições com diferentes objetivos. Por forma a reduzir o impacto dos diferentes tamanhos das estruturas, uma função objetivo usada usualmente considera a média dos desvios da dose prescrita em cada estrutura considerada (Lim et al, 2007a): 


$$
\begin{gathered}
f(D)=\alpha_{P T V} \frac{\| D_{P T V}-T G_{P T V}||_{p}}{\operatorname{card}(P T V)}+\alpha_{O A R} \frac{||\left(D_{O A R}-U B_{O A R}\right)_{+}||_{p}}{\operatorname{card}(O A R)}+ \\
\alpha_{N T} \frac{\left\|\left(D_{N T}-U B_{N T}\right)_{+}\right\|_{p}}{\operatorname{card}(N T)}, \quad p=1,2, \infty,
\end{gathered}
$$

onde $D_{P T V}$ e $T G_{P T V}$ correspondem à dose e objetivo de dose no PTV respetivamente, $D_{O A R}$ e $D_{N T}$ correspondem à dose nos órgãos de risco e tecidos de células normais respetivamente, $\operatorname{card}(\cdot)$ denota o número total de voxeis na estrutura considerada, $p$ a norma considerada e $\alpha_{(\cdot)}$ os pesos de cada estrutura que têm de ser ajustados pelo físico-médico. Diferentes parcelas, correspondendo a diferentes estruturas, podem ser considerados usando diferentes normas na mesma função objetivo. A norma $L_{1}$ penaliza o desvio da dose prescrita, em valor absoluto, em cada voxel, ponderado de acordo com a estrutura ao qual o voxel pertence. A norma $L_{2}$ penaliza a soma dos quadrados dos desvios da dose prescrita, ponderados pelos pesos definidos. A norma $L_{\infty}$ penaliza "pontos quentes" em estruturas sensíveis (OARs e NT) e "pontos frios" no PTV. Note-se que o uso da norma $L_{2}$ origina modelos não lineares quadráticos.

Um revés importante no uso destes modelos de programação linear reside no facto de as soluções ótimas calculadas serem pontos extremos da região admissível, havendo restrições que são satisfeitas como igualdade. Quer isto dizer que, na maior parte das vezes, a solução ótima atribui a dose máxima permitida aos OARs e/Ou o PTV recebe a dose mínima permitida. Caso as restrições sejam demasiado apertadas, por forma a minorar o revés anterior, temos um revés ainda maior: encontrar uma solução admissível pode tornar-se muito difícil ou até mesmo impossível.

Para além dos modelos de programação linear, é também possível considerar modelos não lineares que apresentam como vantagem o facto de garantirem a existência de soluções admissíveis. Considere-se que cada voxel é penalizado de acordo com o quadrado da diferença entre a dose recebida pelo voxel e a dose desejada/permitida para aquele voxel. Esta formulação origina um problema de programação quadrático apenas com restrições de não 
negatividade dos valores das intensidades dos sub-feixes (Romeijn et al, 2003):

$$
\min _{w} \sum_{i=1}^{N_{v}} \frac{1}{v_{S}}\left[\underline{\lambda}_{i}\left(T_{i}-\sum_{j=1}^{N_{b}} D_{i j} w_{j}\right)_{+}^{2}+\bar{\lambda}_{i}\left(\sum_{j=1}^{N_{b}} D_{i j} w_{j}-T_{i}\right)_{+}^{2}\right]
$$

t.q. $\quad w_{j} \geq 0, j=1, \mathrm{~K}, N_{b}$,

onde $T_{i}$ é a dose desejada para o voxel $i, \underline{\lambda}_{i}$ e $\bar{\lambda}_{i}$ são as penalidades de subdosagem e sobredosagem do voxel $i$ e $(\cdot)_{+}=\max \{0, \cdot\}$. Apesar desta formulação permitir pesos diferentes para cada voxel, analogamente à implementação em Aleman et al (2008) podemos atribuir pesos por estrutura por forma a que todos os voxeis de uma dada estrutura tenham os mesmos pesos (dividido pelo número de voxeis da estrutura). Esta formulação não linear implica que uma pequena quantidade de subdosagem ou sobredosagem possa ser aceitável num processo de decisão clínica, mas desvios maiores relativamente à dose desejada/prescrita são decrescentemente tolerados (Aleman et al, 2008).

Do problema de otimização de intensidades irá surgir uma solução que consiste na definição das intensidades de cada sub-feixe para cada direção de incidência. É depois necessário reproduzir de forma o mais fidedigna possível, estas intensidades (contínuas) em intensidades que possam ser realizadas pelo MLC. Tipicamente, as intensidades dos sub-feixes são discretizadas num conjunto de valores ( 0 a 10, e.g.) e uma das muitas técnicas existentes (Alber \& Nusslin, 2001; Men et al, 2007; Romeijn et al, 2005; Shepard et al, 2002; Tervo et $a l, 2003)$ é usada para construir as sequências de posições das folhas do linac. Note-se que a discretização sem critério das intensidades ótimas leva a uma deterioração da qualidade do plano otimizado, que pode e deve ser minorada (Rocha et al, 2014a; Rocha et al, 2014b).

\section{Problema de otimização angular}

Iremos considerar o problema de otimização angular em que pretendemos determinar as direções ótimas de incidência dos feixes de radiação, considerando o número total de ângulos definido a priori. Sendo assim, queremos escolher uma determinada combinação de ângulos, dentro de todas 
as combinações possíveis. A formulação do problema de otimização angular como um problema de programação matemática requer uma medida quantitativa para comparar a qualidade de diferentes combinações de direções de incidência da radiação. A maneira mais correta de se quantificar a qualidade de uma combinação de ângulos terá de ter em conta a solução do problema de otimização de intensidades que se encontra imediatamente a jusante deste problema. Qualquer outra medida de qualidade não oferece qualquer garantia de optimalidade ou fiabilidade, pois tem sido extensamente reportado que direções de irradiação ótimas de tratamentos em IMRT são muitas vezes não intuitivas (Stein et al, 1997). Como tal, a nossa formulação do problema de otimização angular é baseada nas soluções ótimas do problema de otimização das intensidades. Posteriormente, descrevemos o método de resolução por nós sugerido.

\subsection{Formulação do problema de otimização angular}

Consideremos $n$ como sendo o número de direções de irradiação coplanares fixas, i.e., são escolhidos $n$ ângulos num círculo em torno do tumor. Tipicamente, o problema de otimização angular é formulado como sendo um problema de otimização combinatória no qual os $n$ ângulos são selecionados entre um conjunto de ângulos candidatos. Geralmente, o intervalo de ângulos possíveis, $\left[0^{\circ}, 360^{\circ}\right]$, é discretizado em direções equidistantes, com um dado incremento de ângulo (e.g., 5 ou 10 graus). Podemos pensar numa pesquisa global exaustiva de todas as possíveis combinações dos $n$ ângulos. Contudo, isso requer um tempo de cálculo e comparação de todas as diferentes distribuições de dose incompatível com uma resposta num espaço temporal clinicamente aceitável. Por exemplo, escolhendo $n=5$ ângulos entre 72 candidatos, $\quad\left\{0^{\circ}, 5^{\circ}, \ldots, 355^{\circ}\right\} \quad$ existem $C_{5}^{72}=13,991,544$ combinações possíveis. Diminuindo o número de candidatos para $36,\left\{0^{\circ}, 10^{\circ}, \ldots, 350^{\circ}\right\}$, o número de combinações possíveis é ainda de $C_{5}^{36}=376,992$, o que requer um tempo de cálculo de grande magnitude, independentemente da medida usada para comparar os planos de tratamento resultantes. Portanto, uma pesquisa exaustiva num problema combinatório de grande dimensão é considerada 
demasiadamente lenta e inapropriada para uma resposta clínica atempada. Muitas heurísticas e meta-heurísticas têm sido apresentadas como tentativas para reduzir o número de comparações a efetuar. Contudo, a maioria requer um número proibitivo de avaliações da função objetivo, quando a medida usada é o valor ótimo do problema de otimização das intensidades.

A nossa opção consiste em considerar uma aproximação diferente para a formulação do problema de otimização angular. Todos os ângulos contínuos no intervalo $\left[0^{\circ}, 360^{\circ}\right]$ são considerados em vez de uma amostra discreta. Como o ângulo $-5^{\circ}$ é equivalente ao ângulo $355^{\circ}$, e o ângulo $365^{\circ}$ é o mesmo que o ângulo $5^{\circ}$, podemos evitar uma formulação com restrições. Uma formulação para o problema de otimização angular é obtida selecionando uma função objetivo tal que o melhor conjunto de direções ou ângulos de irradiação é obtido para o mínimo da função:

$$
\begin{aligned}
& \min f\left(\theta_{1}, \ldots, \theta_{n}\right) \\
& \text { t.q. }\left(\theta_{1}, \ldots, \theta_{n}\right) \in \mathbb{R}^{n}
\end{aligned}
$$

Aqui, a função objetivo $f\left(\theta_{1}, \ldots, \theta_{n}\right)$ que mede a qualidade do conjunto de direções de irradiação $\theta_{1}, \ldots, \theta_{n}$ é o valor ótimo do problema de otimização das intensidades para o conjunto de direções de irradiação fixo $\theta_{1}, \ldots, \theta_{n}$. Cada conjunto de direções de irradiação fixo $\theta_{1}, \ldots, \theta_{n}$ origina uma matriz de dose $D$ diferente e, consequentemente, um problema de otimização das intensidades com soluções $(w)$ e valores ótimos da função objetivo $f\left(\theta_{1}, \ldots, \theta_{n}\right)$ diferentes. Estas funções têm inúmeros mínimos locais, o que exponencia a dificuldade em obter uma boa solução global. Portanto, a escolha do método de resolução é um aspeto crítico para obter uma boa solução. O uso de métodos com derivadas é bastante comum pelo facto de convergirem muito rapidamente para um ótimo local. A inclusão destes métodos nos softwares dos sistemas de tratamentos para resolução do problema de otimização angular é usual, tal como acontece no software do sistema de tratamento usado na instituição com que colaboramos (IPO Coimbra), mas origina resultados muitos fracos, sendo essa uma das principais razões pelas quais nos dedicamos a este 
problema em particular. A nossa formulação foi essencialmente motivada pela possibilidade de usar uma classe de métodos que, ao contrário dos métodos que usam derivadas, consegue evitar o aprisionamento em mínimos locais, característica que achamos fundamental para atacar com sucesso o problema de otimização angular: métodos de procura em padrão.

\subsection{Métodos de procura em padrão}

Os métodos de procura em padrão são métodos de procura direcional que pertencem à classe mais abrangente de métodos sem derivadas (ver Conn et al, 2009) para uma perspetiva detalhada de métodos de otimização sem derivadas). Os métodos de procura em padrão são métodos iterativos que geram uma sucessão de pontos $\left\{x_{k}\right\}$ usando bases positivas (ou conjuntos geradores positivos) e movem-se numa direção que pode produzir uma redução no valor da função objetivo. Uma base positiva para $\mathbb{R}^{n}$ pode ser definida como um conjunto de vetores não nulos de $\mathbb{R}^{n}$, cuja combinação positiva gera $\mathbb{R}^{n}$, mas nenhum subconjunto próprio o faz.

Uma das principais características das bases positivas, que constitui a principal motivação para o seu uso nos métodos de procura direcional, é que, caso o ponto corrente não seja um ponto estacionário, existe sempre um vetor $v_{i}$ numa base positiva que é uma direção de descida (Davis, 1954), i.e., existe um $\Delta>0$ tal que $f\left(x_{k}+\Delta v_{i}\right)<f\left(x_{k}\right)$. Isto é o cerne dos métodos de procura direcional e em particular dos métodos de procura em padrão.

Os métodos de procura em padrão são métodos iterativos que geram uma sucessão de pontos $\left\{x_{k}\right\}$ não crescente (em termos de valor da função objetivo). Dado o ponto corrente $x_{k}$, em cada iteração $k$, o próximo ponto $x_{k+1}$ é escolhido entre um conjunto finito de candidatos numa dada grelha, definida usando os vetores que formam um conjunto gerador positivo, tentando obter um decréscimo no valor da função objetivo: $f\left(x_{k+1}\right)<f\left(x_{k}\right)$. Estes métodos consideram dois passos em cada iteração. O primeiro passo consiste numa procura finita na grelha, com o objetivo de encontrar um novo ponto que decresça o valor da função objetivo no ponto atual. Este passo, chamado passo de procura, tem a flexibilidade de usar qualquer estratégia, método ou 
heurística, ou tirar partido de particularidades ou conhecimento a priori do problema em mãos, desde que apenas um número finito de pontos da grelha seja testado. Este passo fornece a flexibilidade para uma procura global pois permite pesquisas longe da vizinhança do ponto atual, e influencia a qualidade do minimizante local ou ponto estacionário encontrado pelo método. Se o passo de procura não tiver sucesso, é realizado um segundo passo, chamado passo de sondagem, em torno do ponto atual, com o propósito de melhorar o valor da função objetivo. O passo de sondagem segue regras mais estritas e apela aos conceitos de bases positivas. Neste passo, faz-se uma pesquisa local numa vizinhança da grelha do ponto atual que, para um parâmetro de tamanho de grelha, $\Delta_{k}$, suficientemente pequeno, garante uma redução no valor da função objetivo, caso o ponto atual não seja um ponto estacionário (Alberto et al, 2004). Portanto, se o passo de sondagem falhar, o parâmetro $\Delta_{k}$ deve ser reduzido. A escolha mais comum para atualizar este parâmetro é mantê-lo ou duplicá-lo em iterações com sucesso, e dividir por dois, em caso contrário. Note-se que se o parâmetro de tamanho de grelha inicial for uma potência de 2, $\left(\Delta_{0}=2^{p}, p \in \mathbb{N}\right)$, e o ponto inicial for um vetor de inteiros, usando esta atualização usual do parâmetro do tamanho da grelha, todos os pontos seriam um vetor de inteiros até o parâmetro do tamanho da grelha ser inferior a 1. Esta possibilidade é particularmente interessante para o nosso problema de otimização angular.

$\mathrm{Na}$ abordagem ao problema de otimização angular, é de extrema importância a eficiência em termos de número de avaliações da função objetivo. Portanto, o número de pontos pesquisado no passo de procura deve ser minimalista, e guiado por algum significado físico ou biológico. Por outro lado, quando o passo de procura falha em obter um decréscimo no valor da função objetivo, o passo de sondagem deve ser feito com as direções de procura criteriosamente ordenadas de forma a reduzir ainda mais o número de avaliações da função objetivo (pelo menos nas iterações com sucesso). Recentemente, a eficiência dos métodos de procura em padrão foi melhorada significativamente, reordenando as direções de pesquisa de acordo com indicadores de descida construídos a partir de gradientes simpléticos (Custódio 
\& Vicente, 2007). Posteriormente, no passo de procura, foram incluídos modelos quadráticos de norma de Frobenius mínima, para serem minimizados numa região de confiança, o que pode levar a melhorias significativas (Custódio et al, 2010). Tirando partido da existência de uma implementação dos métodos de procura em padrão, que incorpora as melhorias referidas, usamos uma adaptação da última versão do SID-PSM (Custódio et al, 2010; Custódio \& Vicente, 2007) para a resolução do problema de otimização angular.

Os benefícios do uso dos métodos de procura em padrão na resolução do problema altamente não convexo de otimização angular são ilustrados usando um conjunto de exemplos clínicos de tumores de cabeça e pescoço que são apresentados seguidamente.

\section{Exemplos clínicos de tumores de cabeça e pescoço}

Dois exemplos clínicos de casos de tumores de cabeça e pescoço tratados retrospectivamente no IPOC foram usados para testar a nossa aproximação usando métodos de procura em padrão. Os exemplos selecionados foram sinalizados no IPOC como casos complexos, onde uma irradiação apropriada do tumor e a poupança dos OARs, em particular a poupança das parótidas, demonstrou ser particularmente difícil de obter com os planos de tratamento usuais com 7 ângulos de irradiação equidistantes. As tomografias axiais computorizadas (TACs) dos pacientes com as estruturas delineadas foram exportadas via Dicom RT para um ambiente computacional para investigação em radioterapia de acesso livre (ver Figura 3). Como a região da cabeça e pescoço é uma área complexa onde, e.g., as glândulas parótidas estão usualmente muito próximas ou até sobrepostas ao PTV, uma seleção cuidadosa das direções de incidência da radiação pode ser determinante para obter um plano de tratamentos satisfatório.

A medula e o tronco encefálico são dois dos OARs mais críticos nos casos de tumores de cabeça e pescoço. Trata-se de órgãos em série, i.e., órgãos cuja funcionalidade total fica comprometida mesmo quando apenas uma pequena porção do órgão é danificada. Portanto, se a dose máxima tolerada for excedida, tal pode resultar na perda funcional de todo o órgão. Logo, é de vital 
importância não exceder a dose tolerada prescrita para esse tipo de órgãos. Além da medula e do tronco encefálico, as glândulas parótidas são também OARs importantes. As glândulas parótidas são as maiores das três glândulas salivares. Uma complicação comum devido à irradiação das parótidas é a xerostomia (o termo médico para a boca seca devido à falta de saliva). Isto provoca uma perda acentuada da qualidade de vida dos pacientes submetidos a radioterapia para tratamento de tumores de cabeça e pescoço, causando dificuldade em engolir, perda de apetite, etc. As parótidas são órgãos paralelos, i.e., se apenas uma pequena porção do órgão for danificada, a funcionalidade do resto do órgão pode não ser afetada. A sua dose de tolerância depende fortemente da fração do volume que foi irradiada. Portanto, se apenas uma pequena fração do órgão foi irradiada, a dose de tolerância é muito maior do que se uma fração maior fosse irradiada. Logo, para essas estruturas paralelas, a dose média é geralmente considerada em vez da dose máxima na otimização do planeamento inverso de tratamentos em IMRT.

Em geral, a região da cabeça e pescoço é uma área complexa a tratar em radioterapia devido ao grande número de órgãos sensíveis presentes nesta região (e.g., olhos, mandíbula, laringe, cavidade oral, etc.). Para simplificar, neste estudo, os OARs usados na otimização do planeamento foram limitados à medula, tronco encefálico e glândulas parótidas.

Para estes casos de cabeça e pescoço em estudo, o volume tumoral delineado foi separado em dois com diferentes doses prescritas: PTV1 e PTV2. As doses prescritas para os tumores e as doses de tolerância para os OARs considerados na otimização são apresentadas na Tabela 1.

As glândulas parótidas estão muito próximas ou até mesmo sobrepostas aos PTVs, o que ajuda a explicar a dificuldade em poupar as parótidas. Direções de incidência da radiação adequadas podem ajudar todo o processo de otimização, em particular diminuindo a dose de radiação absorvida pelas parótidas. 


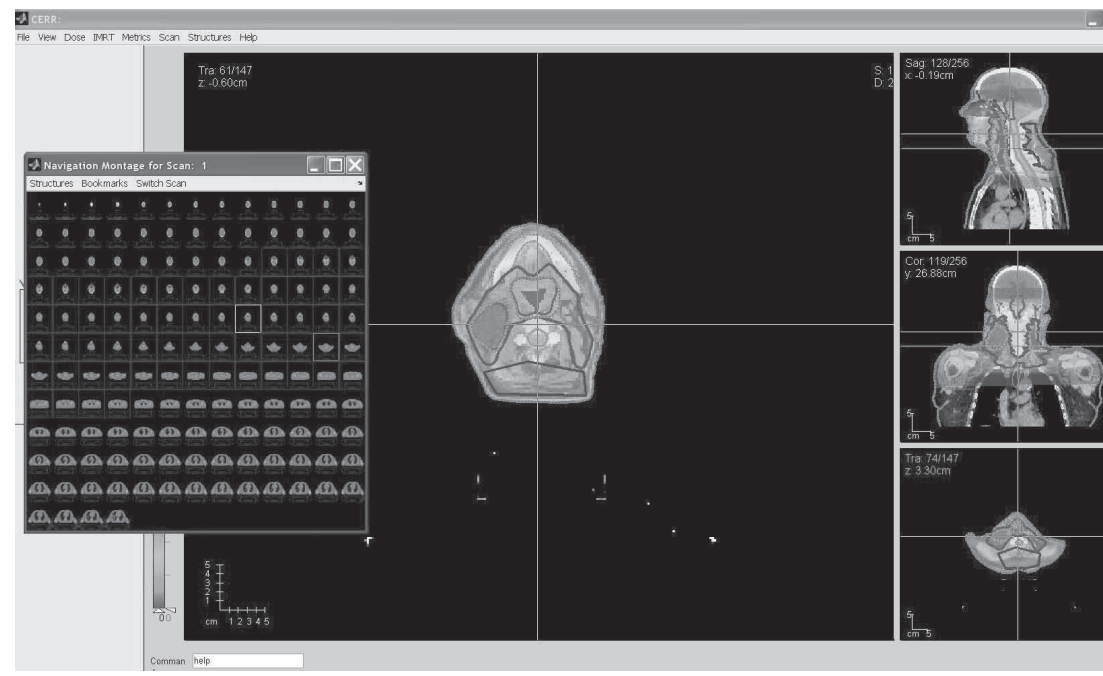

Figura 3: Estruturas consideradas na otimização do planeamento de tratamentos em IMRT.

\begin{tabular}{|c|c|c|c|}
\hline & Dose média & Dose máxima & Dose prescrita \\
\hline Medula & - & $45 \mathrm{~Gy}$ & - \\
\hline Tronco encefálico & - & $54 \mathrm{~Gy}$ & - \\
\hline Parótida esquerda & $26 \mathrm{~Gy}$ & - & - \\
\hline Parótida direita & $26 \mathrm{~Gy}$ & - & - \\
\hline PTV1 & - & - & $70.0 \mathrm{~Gy}$ \\
\hline PTV2 & - & - & $59.4 \mathrm{~Gy}$ \\
\hline Corpo & - & $80 \mathrm{~Gy}$ & - \\
\hline
\end{tabular}

Tabela 1: Doses prescritas e doses de tolerância para todas as estruturas consideradas na otimização do planeamento de tratamentos em IMRT.

\section{Resultados}

Os métodos de procura em padrão aplicados ao problema de otimização angular foram testados usando dois exemplos clínicos de casos de tumores de cabeça e pescoço tratados retrospectivamente no IPOC. A nossa aproximação demonstrou vantagem clara quando comparada com aproximações usando derivadas e estratégias de multistart. Por limitação de espaço, clareza e simplificação da apresentação dos resultados não serão aqui apresentadas essas comparações. Como tarefa futura imediata do projeto de investigação que engloba estes trabalhos de investigação em radioterapia temos a implementação de algoritmos genéticos talhados para o problema de 
otimização angular. A comparação com os resultados obtidos por outros algoritmos que não usam derivadas é também de grande interesse pois, tal como os métodos de procura em padrão, não ficam aprisionados em mínimos locais.

Um plano de tratamento típico, para casos de tumores de cabeça e pescoço, consiste em irradiar o paciente usando entre 5 e 9 direções de incidência da radiação coplanares equidistantes em torno do paciente. Planos de tratamento com 7 direções de incidência da radiação coplanares equidistantes foram usados no IPOC para tratar os pacientes e são usuais na prática clínica para tratamento deste tipo de casos (Aleman et al, 2008). Portanto, foram obtidos planos de tratamento com 7 direções de incidência da radiação coplanares usando métodos de procura em padrão, denotados por 7 PSM, e comparados com os tratamentos usuais com 7 direções de incidência da radiação coplanares equidistantes, denotados por 7 equi. O objetivo desta comparação é demonstrar que a qualidade dos planos de tratamentos podem ser melhoradas usando configurações angulares ótimas obtidas pela nossa aproximação para o problema de otimização angular.

O software de acesso livre CERR - computational environment for radiotherapy research (Deasy et al, 2003) - é usado amplamente para investigação do planeamento de tratamentos em IMRT por facilitar um acesso conveniente aos dados dos planos de tratamento dos pacientes, a sua visualização e análise, bem como permitir a obtenção de dados de dosimetria essenciais para a otimização de planos de tratamento. Este software desenvolvido em MATLAB foi eleito como a plataforma de suporte da nossa otimização do problema inverso do planeamento de tratamentos em radioterapia e serve-nos essencialmente para importar as TACs dos pacientes com as estruturas delineadas para posterior visualização e análise. Tal como referido na secção 2, apesar de o CERR também disponibilizar um algoritmo de Monte Carlo para o cálculo da matriz de dose $D$, por razões de economia de tempo, utilizamos o algoritmo QIB do CERR, menos preciso mas mais rápido (pencil beam). Os nossos testes foram feitos num PC Intel Core Duo com 2.66Ghz de velocidade, usando o MATLAB 7.4.0 (R2007a). Para cada um dos 
dois casos de tumores de cabeça e pescoço, a taxa de sampling usada para o Corpo foi 32 e para as restantes estruturas foi 4, resultando em 21607 (23251) voxeis e 933 (1226) sub-feixes para o plano de tratamentos com 7 direções equidistantes do primeiro (segundo) caso. Foi implementado o cálculo automatizado de dose para cada conjunto de direções de irradiação, em vez do tradicional cálculo de dose usando o módulo IMRTP acessível a partir da barra de opções do CERR. Esta automatização do cálculo da dose foi essencial à integração do nosso algoritmo de otimização angular no CERR.

$\mathrm{Na}$ resolução da nossa formulação não linear convexa do problema de otimização das intensidades usamos um algoritmo de regiões de confiança (fmincon) implementado na Optimization Toolbox do MATLAB 7.4.0. A última versão do SID-PSM foi usada como implementação base dos nossos métodos de procura em padrão. A escolha de pontos iniciais feita de forma aleatória é um procedimento comum, assim como o uso de estratégias de multistart. Como queríamos melhorar a qualidade dos planos de tratamento obtidos usando 7 direções de irradiação equidistantes, consideramos como pontos iniciais no processo de otimização angular exatamente estas configurações angulares equidistantes. A escolha destes pontos iniciais conjuntamente com a propriedade não decrescente da sucessão de pontos gerados pelo SID-PSM implica que cada iteração com sucesso corresponde a uma melhoria efetiva relativamente à configuração equidistante usual. O conjunto gerador positivo usado foi o conjunto ( $\left[\begin{array}{lll}e-e & I-I\end{array}\right]$, sendo $I$ a matriz identidade e $e=\left[\begin{array}{lll}1 & \ldots & 1\end{array}\right]^{T}$ ). Cada uma destas direções corresponde à rotação de todas as direções de incidência da radiação no sentido dos ponteiros do relógio, à rotação de todas as direções de incidência da radiação no sentido contrário ao dos ponteiros do relógio, à rotação individual de cada direção de incidência da radiação no sentido dos ponteiros do relógio e à rotação individual de cada direção de incidência da radiação no sentido contrário à dos ponteiros do relógio, respetivamente. O tamanho da grelha inicial considerado foi 4. Tamanhos de grelha maiores foram testados, nomeadamente 8,16 e 32, sem melhoria dos resultados, e com um aumento significativo do número de avaliações da função objetivo. Como os pontos considerados são vetores inteiros, todos os pontos da 
sucessão gerada pelos métodos de procura em padrão terão valores inteiros enquanto o tamanho da grelha não for inferior a 1 . Portanto, o critério de paragem adotado foi o tamanho da grelha tornar-se inferior a 1.

Uma das principais vantagens dos métodos de procura em padrão é a flexibilidade fornecida pelo passo de procura, onde qualquer estratégia pode ser aplicada desde que apenas um número finito de pontos seja testado. Isto permite a inserção de estratégias/heurísticas previamente usadas com sucesso na resolução do problema de otimização angular, fazendo uma pesquisa global e influenciando a qualidade do mínimo local encontrado pelo método. Na última versão do SID-PSM, o passo de procura calcula um único ponto usando modelos quadráticos com norma de Frobenius mínima a serem minimizados numa região de confiança, o que permitiu uma melhoria significativa na procura direta em funções "black-box" (Custódio et al, 2010) semelhantes às do problema de otimização angular em mãos.

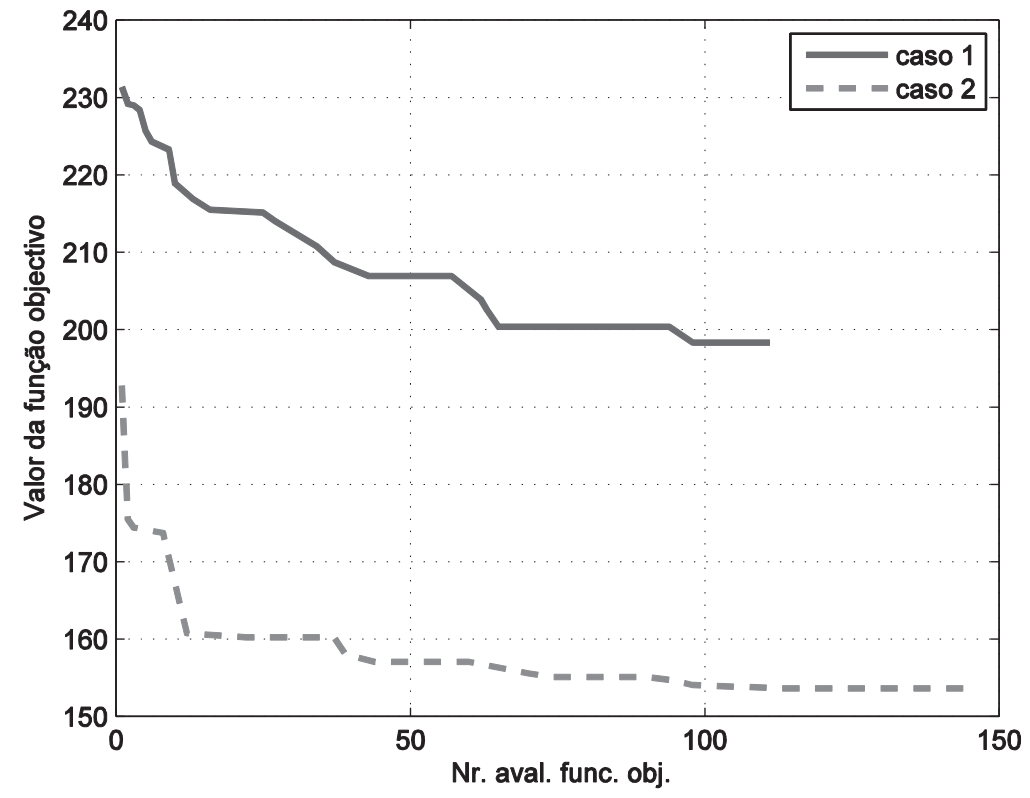

Figura 4: História do processo de otimização angular para cada caso de tumor de cabeça e pescoço em termos do valor da função objetivo.

A história do processo de otimização angular para cada caso de tumor de 
cabeça e pescoço, em termos do valor da função objetivo, usando métodos de procura em padrão, é apresentada na Figura 4. O decréscimo do valor da função objetivo foi em média de 18\% com um tempo computacional médio de 2.6 horas. O cálculo da dose usando o algoritmo QIB do CERR consumiu a maior parte do tempo computacional total. Para diferentes algoritmos de dose, diferentes métodos de otimização das intensidades e diferentes estratégias para a função objetivo, o tempo computacional total pode ter magnitudes significativamente diferentes. Portanto, tão importante quanto mencionar que em média demorou 2.6 horas ao método para convergir, é importante realçar o reduzido número de avaliações da função objetivo requerido pelos métodos de procura em padrão.

Apesar das melhorias do valor ótimo do problema de otimização das intensidades serem um bom indicativo, a qualidade dos resultados pode ser aferida considerando uma variedade de outras métricas. Tipicamente, a qualidade dos resultados é julgada pelos seus histogramas de dose-volume (DVH). O DVH mostra a fração de volume de uma estrutura que recebe pelo menos uma certa dose. Outra métrica usualmente usada para a avaliação de um plano é o volume do PTV que recebe pelo menos 95\% da dose prescrita. Tipicamente, 95\% do volume do PTV é requerido. As doses médias e/ou máximas dos OARs são também usualmente representadas para se verificar se há poupança dos OARs.

Na Figura 5, apresentam-se os DVHs onde é feita a comparação dos planos de tratamento 7 equi com os planos de tratamento 7 PSM. Como as parótidas são os órgãos mais difíceis de poupar, e como os limites das doses máximas da medula e do tronco encefálico foram sempre respeitados, de forma a ilustrar os resultados obtidos o mais nitidamente possível, os DVHs apenas incluem as parótidas e os PTVs. Os asteriscos indicam 95\% do volume dos PTVs versus 95\% das doses prescritas e os círculos indicam a dose média de tolerância para as parótidas. Podemos observar que a irradiação dos tumores em ambos os casos é satisfatória, com as curvas dos PTVs a passarem acima dos asteriscos. Isto significa que, em ambos os casos, os dois planos de tratamento cumprem o objetivo de irradiar pelo menos 95\% do volume dos PTVs com 95\% das doses 
prescritas. Apesar de as irradiações serem muito semelhantes em ambos os planos de tratamento para os dois casos, pode-se verificar uma ligeira vantagem para as curvas obtidas pelo plano de tratamentos 7 PSM, nomeadamente para o segundo caso. Como esperado, as principais diferenças residem na poupança das parótidas. Os planos de tratamento 7 equi não conseguem satisfazer os limites das doses médias das parótidas ao contrário dos planos de tratamento 7 equi. Em média, os planos de tratamento 7 PSM reduzem a dose média nas parótidas em 3 Gy comparativamente aos planos de tratamento 7 equi, facto que é bem ilustrado na Figura 5, o que demonstra a vantagem em usar planos de tratamento com direções de incidência da radiação obtidas pela nossa aproximação em comparação com planos que usam direções equidistantes usadas habitualmente na prática clínica.

É importante salientar que as melhorias obtidas nos planos de tratamento otimizados se refletem numa efetiva melhoria da qualidade de vida dos pacientes. Consideramos que a qualidade dos resultados obtidos pela nossa aproximação é satisfatória quer em termos da melhoria do valor da função objetivo quer em termos da consequente melhoria da qualidade dos planos de tratamento, aferida, e.g., pela poupança das parótidas com uma redução de 3 Gy da dose média comparativamente aos planos de tratamento com direções equidistantes. Contudo, o físico-médico pode não considerar relevante as melhorias obtidas por achar que não refletem uma efetiva melhoria da qualidade dos planos de tratamento oferecido aos pacientes. Isto constitiu uma diferença significativa entre exercícios académicos e este problema real em concreto onde a solução encontrada tem que ser validada. A obtenção de uma solução ótima em termos de valor da função objetivo é por norma o objetivo final de um exercício académico mas, neste problema real, a obtenção de soluções ótimas não leva necessariamente à aceitação do plano de tratamentos resultantes como melhor por parte do físico-médico. Por último, resta realçar que as soluções e correspondentes planos de tratamento obtidos pela nossa aproximação são consideradas pelos físicos-médicos, coautores deste trabalho, como refletindo uma efetiva melhoria relativamente aos planos de tratamento usados habitualmente na prática clínica. 


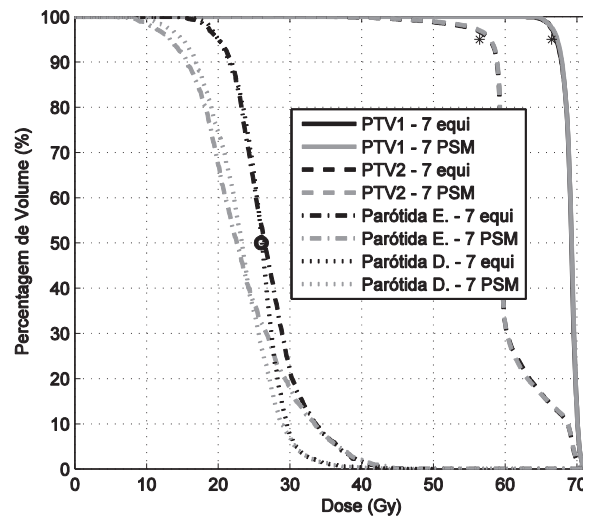

(a)

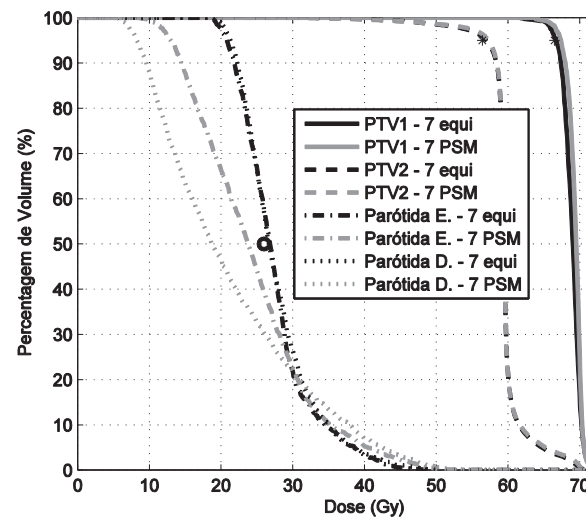

(b)

Figura 5: Histogramas de dose-volume comparando os resultados obtidos por 7 PSM e 7 equi para os casos 1 e 2 , (a) e (b) respetivamente.

\section{Conclusão}

O planeamento de tratamentos em radioterapia, nomeadamente o planeamento inverso, tem tido uma evolução fantástica, desde o uso dos primeiros modelos de otimização usados em radioterapia em 1968, até à atual aplicação clínica generalizada em todo o mundo. A investigação operacional seguiu (e contribuiu para) a evolução das novas máquinas e tecnologias e tem feito contribuições muito relevantes na contínua melhoria do planeamento dos tratamentos em radioterapia. Contudo, novos (e antigos) desafios continuam a necessitar de ser abordados, e este campo de investigação multidisciplinar continua a necessitar da contribuição da investigação operacional para oferecer aos pacientes planos de tratamento com cada vez mais qualidade.

Um dos principais desafios, ainda por resolver de forma satisfatória, é a escolha das melhores direções de incidência da radiação. Na prática clínica, a maioria das vezes, as direções de incidência da radiação continuam a ser escolhidas manualmente pelo físico-médico que confia essencialmente na sua experiência. Tipicamente são necessárias muitas iterações de tentativa e erro entre selecionar as direções de incidência da radiação e calcular as intensidades ótimas dos feixes correspondentes até que se obtenha um plano de tratamentos satisfatório. Este processo é fastidioso, moroso e sem garantias de produzir planos de tratamento de qualidade. Processos automatizados de confiança 
deveriam substituir o físico-médico nesta tarefa e libertá-lo para outras tarefas importantes no processo de planeamento, nomeadamente assegurar a qualidade do mesmo. A investigação operacional tem aqui um papel muito importante e planos de tratamento com qualidade superior aos planos de tratamento equidistantes usualmente usados podem ser obtidos. Demonstramos isso na secção 5 onde os modelos e métodos por nós propostos para a resolução do problema de otimização angular originaram planos de tratamento com maior qualidade relativamente aos planos de tratamento usuais na prática clínica.

Resta ainda muito trabalho a fazer nesta área de investigação aplicada e multidisciplinar e a investigação operacional pode e deve continuar a fazer contributos para a melhoria clínica dos tratamentos em radioterapia. Um dos muitos exemplos ainda por explorar é a otimização das direções de incidência da radiação não coplanares de extrema utilidade em casos de tumores de cabeça.

\section{Bibliografia}

Alber, M., Nusslin, F. (2001). Optimization of intensity modulated radiotherapy under constraints for static and dynamic MLC delivery. Phys. Med. Biol. 46, 3229-3239.

Alberto, P., Nogueira, F., Rocha, H., Vicente, L.N. (2004). Pattern search methods for user-provided points: Application to molecular geometry problems. SIAM J. Optim. 14, 1216-1236.

Aleman, D.M., Kumar, A., Ahuja, R.K., Romeijn, H.E., Dempsey, J.F. (2008). Neighborhood search approaches to beam orientation optimization in intensity modulated radiation therapy treatment planning. J. Global Optim. 42, 587-607.

American Cancer Society. Cancer facts and figures (2010). http://www.cancer.org/acs/groups/content/@epidemiologysurveilance /documents/document/acspc-026238.pdf. Consultado online em 2012-01-11.

Bahr, G.K., Kereiakes, J.G., Horwitz, H., Finney, R., Galvin, J., Goode, K. (1968). The method of linear programming applied to radiation treatment planning. Radiology 91 686-693.

Bortfeld, T. (2006). IMRT: a review and preview. Phys. Med. Biol. 51 363-379.

Carlson, W.D., Morkovin, D. (1959). Time-dose relations for optimal radiation treatment of malignancy with minimal damage to normal tissues. Miss. Valley Med. J. 81 287-288.

Censor, Y. (2003). Mathematical optimization for the inverse problem of intensity-modulated radiation therapy. In: J.R. Palta and T.R. Mackie (Eds.), Intensity-Modulated Radiation Therapy: The State of The Art, American Association of Physicists in Medicine (AAPM), Medical Physics Monograph No. 29, Medical Physics Publishing, Madison, Wisconsin, USA, 25-49.

Conn, A.R., Scheinberg, K., Vicente, L.N. (2009). Introduction to Derivative-Free Optimization. SIAM, Philadelphia. 
Craft, D. (2007). Local beam angle optimization with linear programming and gradient search. Phys. Med. Biol. 52, 127-135.

Craft, D., Halabi, T., Shih, H., Bortfeld, T. (2006). Approximating convex Pareto surfaces in multiobjective radiotherapy planning. Med. Phys. 33, 3399-3407.

Custódio, A.L., Rocha, H., Vicente, L.N. (2010). Incorporating minimum Frobenius norm models in direct search. Comput. Optim. Appl. 46, 265-278.

Custódio, A.L., Vicente, L.N. (2007). Using sampling and simplex derivatives in pattern search methods. SIAM J. Optim. 18, 537-555.

Das, S.K., Marks, L.B. (1997). Selection of coplanar or non coplanar beams using three-dimensional optimization based on maximum beam separation and minimized nontarget irradiation. Int. J. Radiat. Oncol. Biol. Phys. 38, 643-655.

Davis, C. (1954). Theory of positive linear dependence. Am. J. Math. 76, 733-746

Deasy, J.O., Blanco, A.I., Clark, V.H. (2003). CERR: A Computational Environment for Radiotherapy Research. Med. Phys. 30, 979-985.

Deasy, J.O., Lee, E.K., Bortfeld, T., Langer, M., Zakarian, K., Alaly, J., Zhang, Y., Liu, H., Mohan, R., Ahuja, R., Pollack, A., Purdy, J., Rardin, R. (2006). A collaboratory for radiation therapy planning optimization research. Ann. Oper. Res. 148, 55-63

Ehrgott, M., Guler, C., Hammacher, H.W., Shao, L. (2008). Mathematical optimization in intensity modulated radiation therapy. 4OR 6, 199-262.

Ehrgott, M., Johnston, R. (2003). Optimisation of beam directions in intensity modulated radiation therapy planning. OR Spectrum 25, 251-264.

Ferris, M.C., Lim, J.-H., Shepard, D.M. (2003a). Optimization approaches for treatment planning on a Gamma Knife. SIAM J. Optim. 13, 921-937.

Ferris, M.C., Lim, J.-H., Shepard, D.M. (2003b). Radiosurgery treatment planning via nonlinear programming. Ann. Oper. Res. 119, 247-260.

Holder, A., Salter, B. (2004). A tutorial on radiation oncology and optimization. In: H. Greenber (Eds.), Emerging Methodologies and Applications in Operations Research, Kluwer Academic Press, Boston, USA.

Lee, E.K., Fox, T., Crocker, I. (2003). Integer programming applied to intensity-modulated radiation therapy treatment planning. Ann. Oper. Res. 119, 165-181.

Lim, G.J., Ferris, M.C., Wright, S.J., Shepard, D.M., Earl, M.A. (2007a). An optimization framework for conformal radiation treatment planning. INFORMS J. Comput. 19, 366-380.

Lim, G.J., Lee, E.K. (2007b). Optimization in Medicine and Biology. Taylor and Francis, Auerbach Publications, USA

Liu, H.H., Jauregui, M., Zhang, X., Wang, X., Dongand, L., Mohan, R. (2006). Beam angle optimization and reduction for intensity-modulated radiation therapy of non-small-cell lung cancers. Int. J. Radiat. Oncol. Biol. Phys. 65, 561-572.

Men, C., Romeijn, H.E., Taskin, Z.C., Dempsey, J. F. (2007). An exact approach to direct aperture optimization in IMRT treatment planning. Phys. Med. Biol. 52, 7333-7352.

Preciado-Walters, F., Langer, M.P., Rardin, R.L., Thai, V. (2006). Column generation for IMRT cancer therapy optimization with implementable segments. Ann. Oper. Res. 148, 65-79.

RadiologyInfo. http://www.radiologyinfo.org/en/info.cfm?pg=linac. Consultado online em 2012-01-11.

Rocha, H., Dias, J.M. (2009). On the Optimization of Radiation Therapy Planning. Inescc 
Research Reports, nr. 15.

Rocha, H., Dias, J.M., Ferreira, B.C., Lopes, M.C. (2011). Influence of Sampling in Radiation Therapy Treatment Design. In: B. Murgante et al. (Eds.): ICCSA 2011, Lecture Notes in Computer Science, Springer-Verlag, Berlin, 6784 215-230.

Rocha, H., Dias, J.M., Ferreira, B.C., Lopes, M.C. (2014a). Combinatorial optimization for an improved transition from fluence optimization to fluence delivery in IMRT treatment planning. To appear in Optimization. DOI:10.1080/02331934.2011.607498.

Rocha, H., Dias, J.M., Ferreira, B.C., Lopes, M.C. (2014b). Discretization of optimal beamlet intensities in IMRT: a binary integer programming approach. To appear in Mathematical and Computer Modelling. DOI:10.1016/j.mcm.2011.11.056.

Romeijn, H.E., Ahuja, R.K., Dempsey, J.F., Kumar, A. (2006). A new linear programming approach to radiation therapy planning problems. Oper. Res. 54, 201-216.

Romeijn, H.E., Ahuja, R.K., Dempsey, J.F., Kumar, A. (2005). A column generation approach to radiation therapy treatment planning using aperture modulation. SIAM J. Optim. 15, 838-862.

Romeijn, H.E., Ahuja, R.K., Dempsey, J.F., Kumar, A., Li, J. (2003). A novel linear programming approach to fluence map optimization for intensity modulated radiation therapy treatment planing. Phys. Med. Biol. 48, 3521-3542.

Romeijn, H.E., Dempsey, J.F., Li, J. (2004). A unifying framework for multi-criteria fluence map optimization models. Phys. Med. Biol. 49, 1991-2013.

Rowbottom, C.G., Webb, S., Oldham, M. (1998). Improvements in prostate radiotherapy from the customization of beam directions. Med. Phys. 25, 1171-1179.

Shepard, D.M., Earl, M.A., Li, X.A., Naqvi, S., Yu, C. (2002). Direct aperture optimization: a turnkey solution for the step-and-shoot IMRT. Med. Phys. 29, 1007-1018.

Spirou, S., Chui, C.-S. (1998). A gradient inverse planning algoritm with dose-volume constraints. Med. Phys. 25, 321-333.

Stein, J., Mohan, R., Wang, X.H., Bortfeld, T., Wu, Q., Preiser, K., Ling, C.C., Schlegel, W. (1997). Number and orientation of beams in intensity-modulated radiation treatments. Med. Phys. 24, 149160.

Tervo, J., Kolmonen, P., Lyyra-Laitinen, T., Pintér, J.D., Lahtinen, T. (2003). An optimization-based approach to the multiple static delivery technique in radiation therapy. Ann. Oper. Res. 119, 205-227.

Vaz, A.I.F., Vicente, L.N. (2007). A particle swarm pattern search method for bound constrained global optimization. J. Global Optim. 39, 197-219. 